\title{
The experience of a smoke-free policy in a medium secure hospital
}

\author{
Abhijeeth Shetty, ${ }^{1}$ Rajesh Alex, ${ }^{1}$ Darran Bloye ${ }^{2}$
}

The Psychiatrist (2010), 34, 287-289, doi: 10.1192/pb.bp.109.027425

${ }^{1}$ Sheffield Health and Social Care Trust, Sheffield; ${ }^{2}$ Nottinghamshire Health Care Trust, Wathwood Hospital Regional Secure Unit, Rotherham Correspondence to Darran Bloye (Darran.Bloye@nottshc.nhs.uk)
Aims and method A Trust-wide smoke-free policy was introduced in March 2007. This service evaluation retrospectively reviewed the outcome by focusing on recorded changes in behaviour, incidents and prescribing.

Results The majority of patients (89\%) smoked before the smoke-free policy. There was no significant change in the rate of clinical incidents or the use of tranquillising medication at 3 months and 12 months post-implementation. Clozapine serum levels were raised significantly, necessitating dose reduction in $17 \%$ of patients. Fifty-four per cent of patients used nicotine replacement therapy and a small minority continued this treatment for 12 months.

Clinical implications Despite initial concerns there were no significant problems in implementation of the smoke-free policy. This was assisted by extensive preparation, education, patient advocacy and access to treatment beforehand.

\section{Declaration of interest None.}

Tobacco smoking has been estimated to account for up to 5 million deaths worldwide in 2006 and a further 650 million people (or half of the world's smokers alive today) will die prematurely from a tobacco-related disease if they continue to smoke. ${ }^{1}$ Smoking is the greatest cause of morbidity and mortality in the UK, with more than 120000 active and passive smokers dying annually from smoking-related diseases. $^{2}$ About $25 \%$ of the UK adult population smoke and $9 \%$ are heavy smokers, consuming more than 20 cigarettes a day. ${ }^{3}$ Studies of psychiatric in-patients show that up to $70 \%$ smoke, and about $50 \%$ are heavy smokers. ${ }^{4,5}$

The Health Act 2006 introduced legislation that prohibited smoking in all enclosed public areas and workplaces. In-patient mental health units in England and Wales were obliged to ensure that wards and communal areas became smoke-free, and from 1 July 2008 the legislation covered any enclosed or substantially enclosed part of a mental health unit. A survey of 151 mental health units exposed positive attitudes to smoking, with staff respondents describing the benefits of tobacco in terms of relieving boredom and stress, enabling social contact and rapport, and managing aggressive behaviour. ${ }^{6}$ A similar survey undertaken within the Trust showed that more than $80 \%$ of nursing staff believed that patients would become more aggressive, require more medication and display more suicidal behaviours in a smoke-free environment.

\section{Method}

Wathwood Hospital is a 60-bed medium secure unit that admits adult men with primary diagnoses of mental illness.
In-patients are distributed between three wards (assessment, continuing care and rehabilitation) according to levels of risk. Nottinghamshire Healthcare National Health Service (NHS) Trust introduced a smoke-free policy in March 2007 prohibiting the use of tobacco products within the buildings and grounds of all Trust premises. This required all in-patients in medium secure units to abstain from tobacco, except in cases where the clinical team agreed there was a clinical reason not to enforce abstinence (in practice none) or for the small number of patients who had unescorted community leave. The policy was introduced following extensive preparatory work that included smoking cessation groups and individual sessions, introduction of nicotine replacement therapy (NRT), staff training and engagement with patients through posters, individual and group discussion and patient advocates.

We retrospectively evaluated the impact of the policy by reviewing multidisciplinary clinical records, primary healthcare records and incident forms pertaining to the 3month periods before and after implementation, and again 12 months after implementation. Data collection from a sample of all the 56 in-patients resident at the time focused on rates of smoking, incidents of smoking-related verbal and physical aggression, use of as and when needed (p.r.n.) tranquillising medication, clozapine serum levels and use of NRT. Results were analysed using SPSS version 16 for Windows. The Mann-Whitney $U$-test was used to test statistical differences between data before and after implementation of the smoke-free policy, and a $P$ value of $<0.05$ was considered significant. 


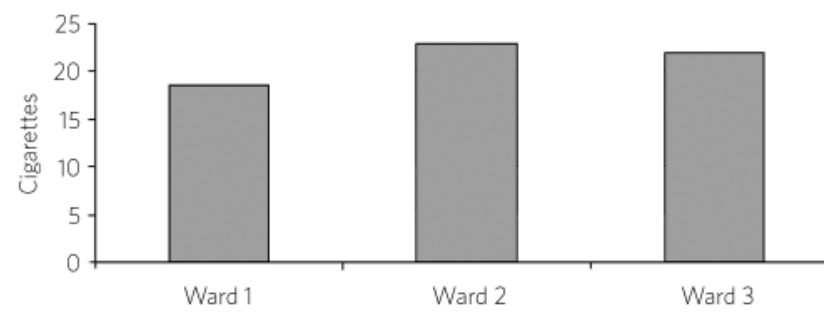

Fig 1 Average daily cigarette consumption prior to implementation of the smoke-free policy (Ward 1, assessment; Ward 2, continuing care; Ward 3, rehabilitation)

\section{Results}

Fifty (89\%) patients smoked prior to the policy and there was evidence to suggest that the average daily consumption of cigarettes increased as patients progressed from the assessment ward to the continuing care ward, decreasing slightly in the rehabilitation ward (Fig. 1). The mean number of cigarettes smoked daily per patient was 21 (range 5-50), reaching the threshold for heavy smoking. A majority of patients objected to the policy and three patients threatened violence to staff or other patients if forced to abstain. Ten patients (20\%) attended a smoking cessation course, seven (14\%) were already contemplating abstinence and two patients gave up smoking prior to the policy implementation.

Twenty-seven (54\%) patients used NRT following policy implementation, some requiring treatment for longer than the 3-month period recommended in local guidelines. Two patients were involved in verbal outbursts attributed to nicotine withdrawal during the first month after policy implementation. None of the patients who threatened violence before the smoke-free policy were involved in any aggressive incident during the follow-up period.

Although there was a reduction in the number of incidents (Table 1), there was no statistically significant change in rates of aggression $(P=0.9$ for verbal aggression (Table 2) and $P=0.6$ for physical aggression) or use of tranquillisers $(P=0.6$ for lorazepam and $P=0.4$ for haloperidol). Twenty-three (41\%) patients received clozapine, all of whom were smokers. There was a significant increase in clozapine levels $(P=0.006)$ and it was necessary to reduce the dose in four (17\%) patients. There was no recorded verbal or physical aggression directly related to nicotine withdrawal 1 year after implementation, but seven patients had contravened the policy by way of illicit use or possession of tobacco. Ten $(20 \%)$ patients were receiving NRT 12 months post-implementation, of whom four had received intermittent nicotine replacement for over 12 months.

\section{Discussion}

Eighty-nine per cent of the patients smoked and most were heavy smokers, which is significantly higher than rates reported in the literature for psychiatric in-patients.,

Table 1 Number of incidents of verbal and physical
aggression

Table 2 Number of patients involved in incidents of verbal aggression

\begin{tabular}{lcc} 
& \multicolumn{2}{c}{ Number of patients } \\
\cline { 2 - 3 } $\begin{array}{l}\text { Number of incidents } \\
\text { per patient }\end{array}$ & $\begin{array}{c}3 \text { months before } \\
\text { smoke-free policy }\end{array}$ & $\begin{array}{c}3 \text { months after } \\
\text { smoke-free policy }\end{array}$ \\
\hline 0 & 46 & 47 \\
\hline $1-5$ & 8 & 9 \\
\hline $6-10$ & 2 & 0 \\
\hline
\end{tabular}

There was a trend of increasing cigarette consumption as the admission progressed and there is evidence to indicate that psychiatric admission can induce non-smokers to smoke. ${ }^{7}$

It has been widely accepted that smoke-free policies are difficult to implement in mental health settings as a consequence of presumed cultural, institutional and clinical factors. ${ }^{8}$ Professionals have traditionally used tobacco as a vehicle to establish rapport with patients and manage challenging behaviour. Nicotine relieves boredom and distress, and facilitates social interaction; however, cigarettes can also be a source of conflict within mental health units, leading to bullying and exploitation.9,10

The minority of non-smoking patients welcomed the policy, and a significant proportion of the patients who smoked engaged in smoking cessation programmes in advance of the ban, recognising the health benefits. The impact of the policy on clinical incidents was less than expected: in the first month, only two patients were involved in verbal aggression directly attributed to nicotine withdrawal, there was no significant change in rates of overall aggression or tranquilliser use and no tobaccorelated aggression was reported 1 year post-implementation. Removing the ward smoking-room, where patients were allowed in one at a time to prevent bullying, and reducing staff time administering cigarettes or lighters, has eliminated a potential source of conflict.

The successful implementation of a smoke-free policy was encouraged by consistent leadership throughout the Trust and careful preparation. There was extensive consultation with staff, patients and advocates, linked to locally delivered smoking cessation programmes. Within our service the physical and procedural security infrastructure was already adapted to the prevention of illicit substance use, and unlike other areas of the Trust we did not face the ethical problem of preventing informally admitted patients who wanted a cigarette from leaving the unit against medical advice. 
Nicotine replacement therapy was used by just over half of the men in our sample. This was an unexpectedly low proportion, although consistent with previous research, ${ }^{11}$ but a significant minority required long-term maintenance. The risk of dependency on NRT is said to be small, with approximately $5 \%$ of abstainers requiring long-term maintenance. ${ }^{12}$ We also observed a significant rise in clozapine serum plasma concentrations, consistent with previous research that showed a $50 \%$ increase in serum levels within 2-4 weeks of abstinence. ${ }^{13}$

The effect of the smoke-free policy has been to place consumption of tobacco on a par with the use of alcohol or illegal drugs. Unsurprisingly, there has been a trade in NRT (particularly lozenges) and a few incidents of illicit use or possession. The long-term impact of a smoke-free policy requires further evaluation and it is disappointing that our anecdotal observations indicate a resumption of binge-type smoking during unescorted leave and heavy consumption among patients reviewed after discharge. Given the implications for clozapine metabolism, it is important that smoking status is communicated to receiving teams. Nevertheless, it is our overall experience that a smoke-free policy can be successfully implemented within a psychiatric hospital without compromising patient safety or increasing clinical risk.

\section{Acknowledgements}

Thanks to Lisa Sutton, Primary Healthcare Manager, Wathwood Hospital Regional Secure Unit, for providing primary healthcare reports and to Pankaj Agarwal, Specialty Registrar, Sheffield Health and Social Care Trust, Sheffield, for assisting in data collection.

\section{About the authors}

Abhijeeth Shetty and Rajesh Alex are Specialist Registrars at Sheffield Health and Social Care Trust, Sheffield. Darran Bloye is Consultant Forensic
Psychiatrist at Nottinghamshire Health Care Trust, Wathwood Hospital Regional Secure Unit, Rotherham.

\section{References}

1 World Health Organization. Tobacco: Deadly in Any Form or Disguise. WHO, 2006 (http://www.who.int/tobacco/communications/events/ wntd/2006/Tfi_Rapport.pdf).

2 Department of Health. Smoke-free Premises and Vehicles. Consultation on Proposed Regulations to be Made Under the Powers in the Health Bill. TSO (The Stationery Office), 2006.

3 Goddard E, Green H. Smoking and Drinking Among Adults, 2004. General Household Survey 2004. Office for National Statistics, 2005.

4 Coulthard M, Farrell M, Singleton N, Meltzer H. Tobacco, Alcohol and Drug Use and Mental Health. TSO (The Stationery Office), 2002.

5 Kelly C, McCreadie RG. Smoking habits, current symptoms and premorbid characteristics of schizophrenic patients in Nithsdale, Scotland. Am J Psychiatry 1999; 156: 1751-7.

6 Jochelson K, Majrowski W. Clearing the Air: Debating Smoke-Free Policies in Psychiatric Units. King's Fund, 2006.

7 Lawn SJ, Pols RG, Barber JG. Smoking and quitting: a qualitative study with community-living psychiatric clients. Soc Sci Med 2002; 54: 93104.

8 Jochelson K. Smoke-free legislation and mental health units: the challenges ahead. Br J Psychiatry 2006; 189: 479-80.

9 Lawn SJ. Systemic barriers to quitting smoking among institutionalised public mental health service populations: a comparison of two Australian sites. Int J Soc Psychiatry 2004; 50: 204-15.

10 Hempel AG, Kowanacki R, Malin DH, Ozone SJ, Cormack TS, Sandoval $B G$, et al. Effect of a total smoking ban in a maximum security psychiatric hospital. Behav Sci Law 2002; 20: 507-22.

11 Lawn S, Pols R. Smoking bans in psychiatric in-patient settings? A review of the research. Aust N Z J Psychiatry 2005; 39: 866-85.

12 Molyneux $A$. ABC of smoking cessation: nicotine replacement therapy. BMJ 2004; 328: 454-6.

13 De Leon J. Atypical antipsychotic dosing: the effect of smoking and caffeine. Psychiatr Serv 2004; 55: 491-3. 